\title{
Investigating Disease Spread between Two Assessment Dates with Permutation Tests on a Lattice
}

\author{
Gaël Thébaud, Nathalie Peyrard, Sylvie Dallot, Agnès Calonnec, and Gérard Labonne
}

First, third, and fifth authors: Institut national de la recherche agronomique (INRA), UMR BGPI, CIRAD TA 41/K, Campus international de Baillarguet, 34398 Montpellier Cedex 5, France; first and second authors: INRA, Unité de Biométrie, Domaine Saint-Paul, Site Agroparc, 84914 Avignon Cedex 9, France; and fourth author: INRA, UMR Santé Végétale, 71 avenue Edouard Bourlaux, BP 81, 33883 Villenaved'Ornon Cedex, France.

Accepted for publication 24 August 2005.

\begin{abstract}
Thébaud, G., Peyrard, N., Dallot, S., Calonnec, A., and Labonne, G. 2005. Investigating disease spread between two assessment dates with permutation tests on a lattice. Phytopathology 95:1453-1461.

Mapping and analyzing the disease status of individual plants within a study area at successive dates can give insight into the processes involved in the spread of a disease. We propose a permutation method to analyze such spatiotemporal maps of binary data (healthy or diseased plants) in regularly spaced plantings. It requires little prior information on the causes of disease spread and handles missing plants and censored data. A Monte Carlo test is used to assess whether the location of newly diseased plants is independent of the location of previously diseased plants. The test takes account of the significant spatial structures at each date in order to separate nonrandomness caused by the structure at one date from nonrandomness caused by the dependence between newly diseased plants
\end{abstract}

ABSTRACT and previously diseased plants. If there is a nonrandom structure at both dates, independent patterns are simulated by randomly shifting the entire pattern observed at the second date. Otherwise, independent patterns are simulated by randomly reallocating the positions of one group of diseased plants. Simulated and observed patterns of disease are then compared through distance-based statistics. The performance of the method and its robustness are evaluated by its ability to accurately identify simulated independent and dependent bivariate point patterns. Additionally, two realworld spatiotemporal maps with contrasting disease progress illustrate how the tests can provide valuable clues about the processes of disease spread. This method can supplement biological investigations and be used as an exploratory step before developing a specific mechanistic model.

Additional keywords: censoring, distance class, European stone fruit yellows, nonparametric, Plum pox virus, toroidal shift.
One of the main goals of plant disease epidemiologists is to understand the underlying processes of epidemics. When the epidemiology of a disease is poorly known, for example in the case of an emerging disease $(2,37)$, the initial questions often include the following. How to summarize the progress of the disease in time and space? Is there secondary spread at the scale considered, i.e., do previously diseased plants provide the inoculum that infects the newly diseased plants? What are the biological processes responsible for disease spread? Answering these questions can help identify control strategies, design sampling procedures (21, 23), or build mechanistic models (15).

Understanding plant disease epidemiology was initially based on the analysis of the temporal progress of diseases (41), with the introduction of correction factors (basically, a reduction of the rate of disease progress) to take into account the effect of disease clustering on temporal dynamics $(5,25,42)$. However, temporal analysis has limitations when it is used to draw conclusions about the underlying processes of disease spread because many different phenomena can generate the same temporal progress $(4,5)$. Recently, the spatial patterns of plant diseases have been investigated more systematically. Plant species whose health status can be mapped individually are frequently grown in regular lattices (e.g., orchards, vineyards, some vegetable crops, and commercial forest plots). This article focuses on the exploratory analysis of such

Corresponding author: G. Thébaud; E-mail address: thebaud@ensam.inra.fr

\section{DOI: 10.1094/PHYTO-95-1453}

This article is in the public domain and not copyrightable. It may be freely reprinted with customary crediting of the source. The American Phytopathological Society, 2005. maps as an initial step in epidemiological studies. Because it requires few assumptions and little prior knowledge of the processes of spread, the nonparametric approach based on distances between mapped individuals $(10,36,40)$ is well suited to such preliminary epidemiological studies. It was popularized in plant disease epidemiology by Gray et al. (19), who developed a specific two-dimensional method to identify the spatial patterns of diseased individuals using multiple Monte Carlo tests. Subsequently, several specific software packages have been developed, including 2DCLASS (29), STCLASS (27,28), and 2DCORR (13), which have been used to analyze spatial patterns of plant diseases $(17,18,32,38)$. Spatial analyses not only identify disease clustering, but can also suggest processes of disease spread (a directional effect along rows, for example, often indicates a spread by way of cultural interventions or plant contacts). However, when disease is significantly clustered, several explanations can be given: within-field spread from previously diseased plants, of course, but also proximity to an external source of inoculum (e.g., along field borders), local heterogeneity in plant susceptibility, successive inoculations (for vector-borne diseases with persistent transmission), or planting of a batch of infected plants (19). Multiple interpretations of clustering allow few deductions about the biological processes that are responsible for the observed spatial pattern, mainly due to a possible confounding between spatial and temporal effects (39). Therefore, additional information is necessary to gain insight into the processes of disease spread.

The spatiotemporal pattern of diseased plants can be used to go one step further because it provides a way to distinguish the basic spatial pattern of the disease from its temporal progress. Then it becomes possible to test hypotheses that are more directly related 
to the biological processes of spread. Are the newly diseased plants located at random? Is there a spatial relationship between newly diseased plants and previously diseased plants? Nelson (28) provided a technique to answer the first question using a permutation test in which the positions of missing plants and previously diseased plants are fixed during the simulations of the null hypothesis. However, in his approach to the second question, randomness of disease increase is tested globally: nonrandomness caused by the spatial relationship between newly diseased plants and previously diseased plants is not separated from nonrandomness caused by the spatial structure within newly diseased plants. Mugglestone et al. (26) developed a test of independence between diseased plants at two dates on a lattice, restricted to the situation where diseased plants at the first assessment date are scarce and located at random.

The aim of the work presented here is to develop a nonparametric method to assess whether the location of newly diseased plants depends on the location of previously diseased plants within regular plantings. At each date, diseased plants can be clustered or not, and this information is taken into account: the expected patterns under the null hypothesis are simulated consistently with the observed spatial pattern within each group. Thus, the method is based on a set of three Monte Carlo tests, corresponding to different combinations of patterns at time 1 and time 2. After presenting these tests, their accuracy and robustness are evaluated with computer-simulated data sets, and they are applied to realworld spatiotemporal disease maps.

\section{MATERIALS AND METHODS}

Overview. The method described here is dedicated to the analysis of a common kind of binary data sets that are collected when diseased and healthy plants are mapped over time, and when some plants may be missing for a reason unrelated to the disease. The systems have the following characteristics: (i) plants are regularly spaced along and across rows, but the distance between two plants along rows can differ from the distance across rows; (ii) a plant must be either missing, healthy, diseased at time 1, or diseased at time 2; and (iii) diseased plants at time 1 are still diseased at time 2 (successive inoculations of the same plant cannot be observed; roguing followed by replanting is not taken into account). These features correspond primarily to systemic diseases (5) of vegetables, vines, fruit trees, and commercial forest trees. However, our method can also be used in the analysis of other pathosystems that can reasonably be reduced to a binary spatiotemporal data set (quantitative data transformed into binary data, batch analyses, or a field divided into a grid of contiguous diseased or healthy quadrats).

The tested null hypothesis $\left(\mathrm{H}_{0}\right)$ is as follows: "the location of diseased plants at the second assessment date is independent of the location of previously diseased plants." The value of a statistic measuring the spatial dependence between these two groups of plants is compared with its distribution under $\mathrm{H}_{0}$. To this aim, a set of expected patterns is simulated by randomizing the pattern observed at one date without altering its significant spatial structures. A crucial rule in the simulations is that the probability for a

TABLE 1. Three tests of independence to investigate disease spread on a lattice between two assessment dates, with a decision rule depending on the pattern of diseased plants at each assessment date

\begin{tabular}{lcc}
\hline & \multicolumn{2}{c}{${\text { Pattern of } t_{1} \text { cases }^{\mathrm{b}}}^{\text {Pattern of } t_{2} \text { cases }^{\mathrm{a}}}$} \\
\cline { 2 - 3 } $\begin{array}{l}\text { Random } \\
\text { Structured }\end{array}$ & Random & Structured \\
& Test 1 (permutation of $t_{2}$ cases) \\
& Test 2 (permutation & Test 3 (toroidal shift \\
of $t_{1}$ cases) & of $t_{2}$ cases) \\
\hline
\end{tabular}

\footnotetext{
${ }^{a}$ Diseased plants at the second assessment date.
}

${ }^{\mathrm{b}}$ Diseased plants at the first assessment date. given plant to be in a given status must be the same in simulated data as it had been in the observed data (this implies, for example, that a diseased plant in an expected pattern must not appear at the location of a missing plant in the actual data).

Observed and simulated patterns are then compared through distance class analysis: in both observed and simulated data, every pair of plants composed of one $t_{1}$ case and one $t_{2}$ case is assigned to a distance class $\left(t_{1}\right.$ cases denote diseased plants at time 1 , and $t_{2}$ cases denote healthy plants at time 1 that are diseased at time 2). This class is exclusively determined by the distance between plants, regardless of orientation. The subsequent statistical test is based on the radial cumulative frequency of distances between $t_{1}-t_{2}$ pairs of cases (i.e., the number of $t_{1}-t_{2}$ pairs of cases within a circle of increasing radius): using this cumulative function $(13,22,36)$ instead of a noncumulative function increases the power of the test for the initial distance classes and reduces the variability of the curves. Then, a test statistic is defined to provide a direct evaluation of the cumulative departure of the observed pattern from $\mathrm{H}_{0}$. To assess the significance of this departure, we compute the same statistic on the observed data and on 1,000 Monte Carlo simulations of $\mathrm{H}_{0}$ (which preserves the identified spatial structures - or their absence-within each group). For each distance class, once sorted, the simulated values with a rank corresponding to the upper and lower significance thresholds define a confidence interval for the statistic under $\mathrm{H}_{0}$. In addition, for this bilateral test, an approximate $P$ value is computed, following Manly (24), as twice the proportion of simulated values more extreme than or equal to the observed value.

For a given distance class (and a given significance level, e.g., $5 \%$ ) the test can provide three different results: (i) the number of pairs is too high to be compatible with the independence between $t_{1}$ cases and $t_{2}$ cases; (ii) the number of pairs is too low to be compatible with the independence between $t_{1}$ cases and $t_{2}$ cases; and (iii) we cannot exclude the possibility that $t_{1}$ cases and $t_{2}$ cases result from independent causes. Here, we always assume that nonrandomness can be caused either by an excessive aggregation or by an excessive regularity; thus, we use a two-sided test with a global 5\% significance divided in two symmetrical parts. Of course, if an excess of regularity is impossible, then a one-sided test should be performed with a $5 \%$ significance level. We also applied these general principles to implement directional tests along and across rows, the only difference being that the computed distances only involve plants that are located within the same row (or inter-row).

To test the independence conditional on the intra-group spatial structures, the most general test preserves the pattern within both $t_{1}$ cases and $t_{2}$ cases. However, the systematic inclusion of a spatial structure that may not exist can reduce the power of the test. To avoid such a situation, we suggest taking into account the pattern of $t_{1}$ cases or $t_{2}$ cases only when they are significantly structured (Table 1): when both patterns are structured, $t_{2}$ cases are randomly shifted relative to $t_{1}$ cases (Test 3 ); when only one of the two patterns is significantly structured, the nonstructured pattern is randomized (Test 1 and Test 2); and when none of the two patterns is significantly structured, $t_{2}$ cases are randomized (Test 1). Hence, in the absence of prior knowledge of the expected spatial patterns, a preliminary analysis of the spatial pattern at each date is required to decide which kind of randomization should be applied (and to which set of points). This analysis should be carried out with a method that correctly handles missing plants; if permutations are used, the location of $t_{1}$ cases should be fixed during the random permutations of $t_{2}$ cases. Subsequent to this preliminary analysis, one of the following three tests can be applied.

Random pattern at date 2 (Test 1). Test 1 is performed when the preliminary analysis indicates that the spatial repartition of newly diseased plants ( $t_{2}$ cases) shows no significant departure from randomness, whatever the spatial pattern of $t_{1}$ cases. As in 
STCLASS (28), the locations of both missing plants and $t_{1}$ cases are fixed, and $t_{2}$ cases are randomly reallocated among the other plants. But instead of considering the distances between all diseased plants, we compute a test statistic that is only based on the distances between $t_{1}$ cases and $t_{2}$ cases. Since the representation of cumulative frequencies leads to graphical scaling effects that can mask a significant departure from randomness in the first distance classes (3), counts are standardized by the mean of simulated values, leading to

$$
Q_{c}(d)=\sum_{\delta \leq d} N(\delta) / \sum_{\delta \leq d} \overline{N \operatorname{sim}(\delta)}=N_{c}(d) / \overline{N_{c} \operatorname{sim}(d)}
$$

$N_{c}(d)$ being the number of $t_{1}-t_{2}$ pairs of cases closer than or equal to distance $d$, and $\overline{N_{c} \operatorname{sim}(d)}$ being the mean number of simulated $t_{1}-t_{2}$ pairs of cases closer than or equal to distance $d$.

If the observed value of $Q_{c}(d)$ in a given distance class is significantly different from the simulated set of values (strictly higher than the 975th value or strictly smaller than the $25 \mathrm{th}$, for a bilateral test with a 5\% significance level and 1,000 simulations), we can conclude that the location of $t_{2}$ cases depends on the location of $t_{1}$ cases, i.e., disease did not spread randomly.

Structured pattern at date 2 only (Test 2). Test 2 is performed when $t_{2}$ cases are significantly structured, whereas no spatial structure could be identified within $t_{1}$ cases. This test involves random permutations (reallocations) of $t_{1}$ cases among all plants except missing plants. Thus, some simulated $t_{1}$ cases can overlay $t_{2}$ cases. This is impossible in real spatiotemporal surveys where multiple infections of the same plant cannot be detected ( $t_{1}$ cases hide future potential $t_{2}$ cases; thus, no plant will belong both to $t_{1}$ cases and $t_{2}$ cases in the observed data). The principles of permutation tests require dealing with the resultant censoring. Hence, $t_{2}$ cases on which $t_{1}$ cases are reallocated after a simulation round must be excluded, as demonstrated in the Appendix; such $t_{2}$ cases are not taken into account in the computation of the distances on both observed and simulated data. As each randomization leads to the exclusion of different $t_{2}$ cases, the test statistic has been adapted from usual permutation tests $(6,33)$. Let $\phi$ be a given permutation of $t_{1}$ cases among all plants excluding missing plants, let $Y_{1}$ be the set of $t_{1}$ cases in the actual data, $Y_{1, i}{ }^{\phi}$ the set of $t_{1}$ cases after the $i$ th permutation, and $R_{2, i}$ the subset of $t_{2}$ cases in the actual data that are not censored by $t_{1}$ cases after the $i$ th permutation. After each simulation, we compute the distances between noncensored observed $t_{2}$ cases and either the simulated $t_{1}$ cases or the observed $t_{1}$ cases. The associated functions are $C_{i}^{\phi}(d)=f\left(R_{2, i}\right.$, $\left.Y_{1, i}{ }^{\phi}, d\right)$ and $C_{i}(d)=f\left(R_{2, i}, Y_{1}, d\right)$, respectively, where $f$ denotes the cumulative number of cases closer than or equal to distance $d$. The test is based on the difference between these functions computed on simulated and observed data. As the variability of $C_{i}^{\phi}(d)-$ $C_{i}(d)$ increases along with the distance $d$, it is divided by $d$ to improve the graphical display by an appropriate scaling (which does not affect the result of the test), leading to

$$
\mathrm{S}_{c, i}(d)=\left[C_{i}^{\phi}(d)-C_{i}(d)\right] / d
$$

If $t_{1}$ cases and $t_{2}$ cases are independent, the expected value of the test statistic, $S_{c, i}(d)$, is equal to 0 ; thus, for each distance class $d$, the whole set of values of $S_{c, i}(d)$ is compared with 0 . For a bilateral test with a significance level of $\alpha=5 \%$, if all of the lowest $97.5 \%$ values are negative at a given distance, there is an excess of pairs of diseased plants in the observed data for this distance class. Symmetrically, if the highest $97.5 \%$ values are all positive at a given distance, there is a lack of pairs of diseased plants. Both situations indicate a spatial relationship between $t_{1}$ cases and $t_{2}$ cases (either a positive or a negative association).

Structured patterns at both date 1 and date 2 (Test 3). Test 3 is performed when both $t_{1}$ cases and $t_{2}$ cases are significantly structured to test the independence between the whole pattern of $t_{1}$ cases and the whole pattern of $t_{2}$ cases. This spatiotemporal test is based on Lotwick and Silverman's test (22), adapted following a method to cope with the censoring $(7,33)$ caused by missing plants and $t_{1}$ cases. In order to preserve the existing spatial structures, the permutations that are applied to $t_{2}$ cases are restricted to shifts of the whole set of $t_{2}$ cases. As in the original paper by Lotwick and Silverman (22), the torus convention is used to connect the opposite edges of the plot, with the result that all distances are measured on this torus. Thus, for practical reasons, only rectangular plots can be used (excluding data located beyond the largest rectangular subset from the spatiotemporal map). We show on a hypothetical disease map (Fig. 1A) the initial steps for performing Test 3 . A toroidal shift by a number of plants along and across rows (denoted $\phi$ ) can be applied to the whole map (Fig. 1B). In the test, the observed positions of $t_{1}$ cases are preserved, whereas the whole set of $t_{2}$ cases is shifted on the torus. As in the previous test, some of the shifted $t_{2}$ cases are censored and some of the observed $t_{2}$ cases (overlaid by the shifted censoring pattern) have to be excluded in order to conserve the same distribution of distances in observed and simulated patterns (Appendix and Fig. 1C). Thus, only the points that belong to $R_{2, i}^{\prime}{ }^{\phi}$ and $R_{2, i}^{\prime}$ are used in the calculation of the number of $t_{1}-t_{2}$ pairs of cases closer than or equal to distance $d . R_{2, i}^{\prime}{ }^{\phi}$ denotes the subset
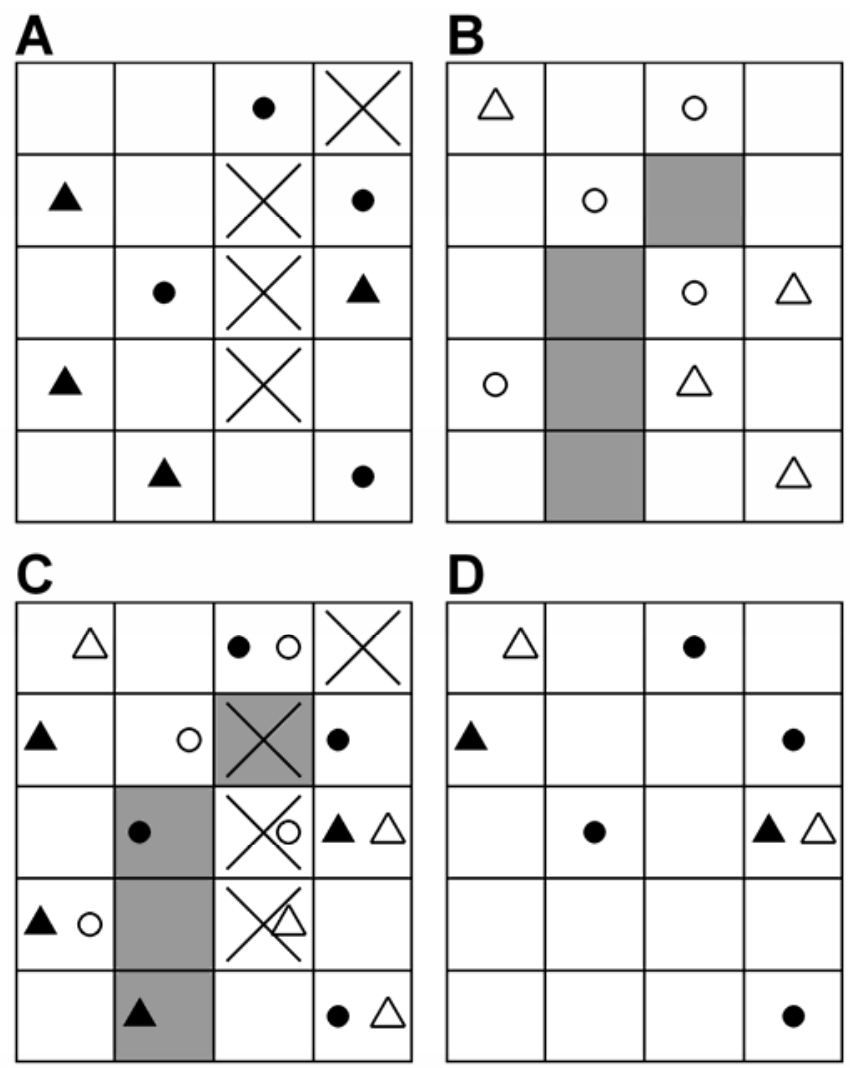

D
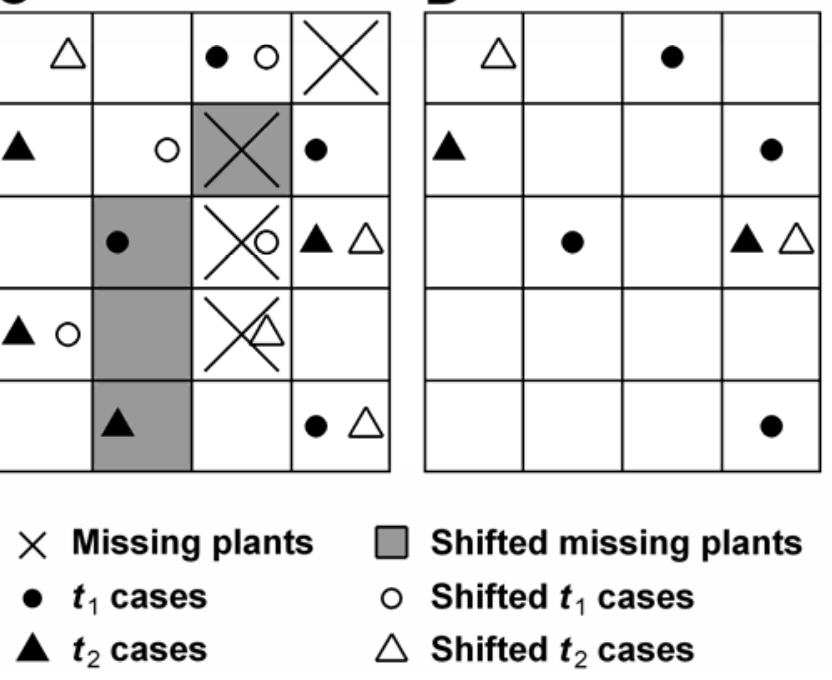

Fig. 1. Handling censoring patterns caused on newly diseased plants ( $t_{2}$ cases) by missing plants and previously diseased plants ( $t_{1}$ cases) in the test based on toroidal shifts (Test 3 ). The observed pattern (A) is randomly shifted (B) on a torus (1 unit to the bottom and 1 unit to the left, in this example). The superposition of observed and shifted patterns $(\mathbf{C})$ excludes some $t_{2}$ cases and results in the final sets of selected points (D). The excluded points are observed $t_{2}$ cases censored by the shift of either a missing plant or a $t_{1}$ case; symmetrically, any shifted $t_{2}$ case censored by an observed missing plant or $t_{1}$ case is excluded. The distances between all $t_{1}$ cases and the subsets of observed and shifted $t_{2}$ cases that have not been censored during the shift are used to compute the test statistic $S_{c, i}(d)$. 
of shifted $t_{2}$ cases that are not censored (by observed $t_{1}$ cases or observed missing plants) after the $i$ th shift (Fig. 1D, open triangles). $R_{2, i}^{\prime}$ is symmetrically defined as the subset of observed $t_{2}$ cases that are not censored (by shifted $t_{1}$ cases or shifted missing plants) after the $i$ th shift (Fig. 1D, filled triangles). The associated functions are $C_{i}^{\prime}{ }_{i}(d)=f\left(Y_{1}, R_{2, i}^{\prime}, d\right)$ and $C_{i}^{\prime}(d)=f\left(Y_{1}, R_{2, i}^{\prime}, d\right)$.

The remaining steps of this test are similar to what has been described above (repeated computation of $S_{c, i}(d)$ and comparison with 0), except for one detail: the maximum number of different shifts is the product of the number of rows by the number of plants by row. Thus, the total number of possible toroidal shifts is much lower than the total number of possible permutations, which can result in a lack of power for small lattices. When the number of plants is lower or slightly above the predefined number (e.g., 1,000) of expected patterns to simulate, all possible shifts are performed one after another rather than performing random toroidal shifts. Doing so leads to an exact test instead of an approximation and can also reduce the computing time required. All the tests were programmed and performed using the statistical $\mathrm{R}$ language (34). The source code is available upon request to the corresponding author.
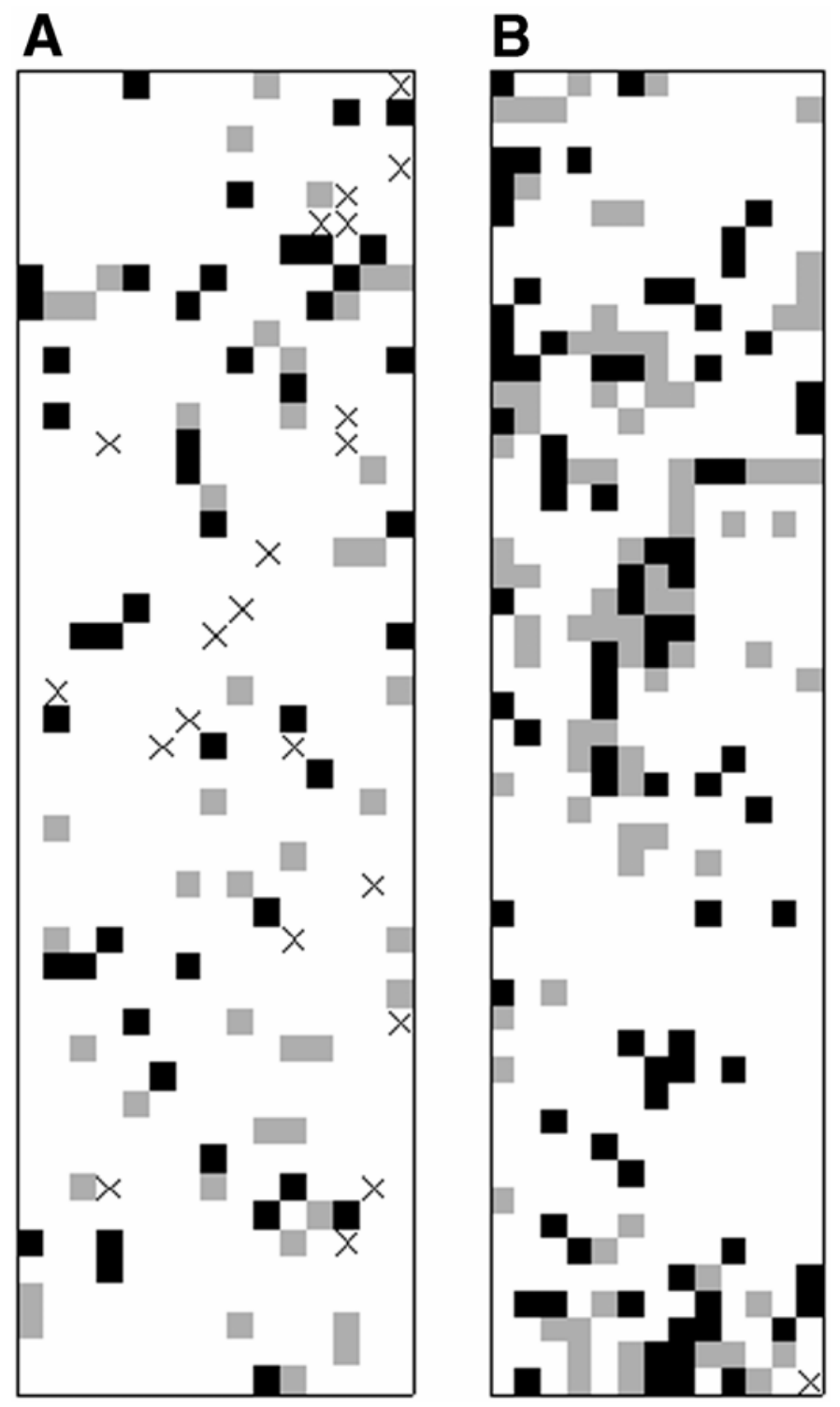

Fig. 2. Spatiotemporal pattern of diseased trees in two orchards affected by A, European stone fruit yellows, and $\mathbf{B}$, Plum pox virus. In both orchards, white, black, and gray squares represent healthy trees, initial infections ( $t_{1}$ cases), and later infections ( $t_{2}$ cases $)$, respectively. Crosses symbolize missing trees.
Numerical validation. A simulation study was performed to evaluate the type I error and the power of the method, as well as its robustness to some deviation from key assumptions. In this evaluation, the first four distance classes were considered. The proportion of 1,000 virtual patterns that is rejected by the test is an estimate of the true level of type I error when independent patterns are simulated; it estimates the statistical power when dependent patterns are simulated. These patterns were generated on a lattice as follows: (i) two independent Poisson processes for the independence between two random patterns, which could correspond to two waves of independently incoming vectors; (ii) two independent Neyman-Scott processes for the independence between two clustered patterns, which could correspond to two independent waves of incoming vectors causing primary infections of the persistent mode; (iii) a Poisson process giving rise to an eight-neighbor contact process for the dependence between two random patterns, which could correspond to a short-distance secondary transmission of the nonpersistent mode after random primary infections; and (iv) a Neyman-Scott process giving rise to a secondary Neyman-Scott process for the dependence between two clustered patterns, which could correspond to multifocal epidemics.

To evaluate the robustness of the method to some deviation from its assumptions, we also simulated the following: (i) $t_{1}$ patterns dependent on missing plants by a Neyman-Scott process using missing plants as centers, and (ii) a border-effect by an inhomogeneous Neyman-Scott process with the distance between each center and the left side of the lattice following an exponential distribution with mean one-third of the lattice width.

For all patterns, the positions of missing plants were assigned at random. For random patterns, the average number of $t_{2}$ cases was half that of $t_{1}$ cases; for clustered patterns, $t_{2}$ cases were twice the number of $t_{1}$ cases, on average. To avoid edge-effects, a 10-plantwide outer margin was added to the observation window before simulating Neyman-Scott processes. For each Neyman-Scott process, cluster centers were located at random (the probability for each position to be a center being 0.013 ), and marked points were created around these centers at a distance following an exponential law with mean 2.5. Unless otherwise stated, the simulations were performed on a 50 by 20 observation window $(1 \times 1$ unit lattice) with a total of $20 \%$ disease incidence and with $2 \%$ missing plants.

Experimental data sets. To illustrate the method, we analyzed data sets from two different vector-borne diseases showing contrasting spatiotemporal patterns. The first data set (Fig. 2A) is a map of trees affected by European stone fruit yellows (ESFY) in an apricot orchard of 720 trees ( 15 rows of 48 trees each), planted in 1977 on a $4 \times 4 \mathrm{~m}$ lattice. Typical symptoms of this phytoplasma disease (e.g., off-season growth, yellowing, and leaf roll) were recorded twice a year, starting in 1983; by 1994, 91 trees had expressed symptoms and 21 were missing for reasons unrelated to ESFY (mainly after showing symptoms of bacterial canker). To test the independence between early and late infections, we made two groups of plants on the basis of the temporal evolution of annual incidence: $t_{1}$ cases expressed symptoms before 1988 and $t_{2}$ cases expressed symptoms between 1989 and 1994.

The second data set (Fig. 2B) is from a peach orchard affected by Plum pox virus strain M (PPV-M). The 663 trees (13 rows of 51 trees each) were planted in 1989 on a $2 \times 5$ m lattice (within rows and between rows, respectively). Each tree was inspected visually for specific PPV symptoms (e.g., vein clearing and mosaic) from 1992 to 1994. By the end of 1994, 169 trees had shown symptoms. Symptomatic trees from the first two assessment dates were pooled in the analyses because not all trees had been correctly examined for PPV symptoms on the first assessment.

The preliminary identification of spatial patterns within each group of cases in the ESFY- and PPV-infected orchards was performed using radial correlation analysis (13), which is designed 
specifically for discrete data; for the analysis at the second assessment date, $t_{1}$ cases were encoded as missing plants. When using the tests presented in this article, we always defined the size of the distance classes as the minimal distance between the trees.

\section{RESULTS}

Simulated data sets. More than 25,000 simulated patterns were tested to determine the performance of the test in various situations. As shown in Table 2, for all the simulated stationary patterns, the true level of type I error is approximately equal to the predefined 5\% level. Despite the censoring of some observations, the power of the test is very high $(>90 \%)$ and increases at the second distance class because of the cumulative nature of the test. For low disease incidence, the test has slightly less power and is somewhat conservative because of ties (the risk to wrongly reject the hypothesis of independence is then $<5 \%$ ). Table 3 shows that the proportion of missing plants has a minor impact on the power of the test (at least up to 10\%), whereas a decreasing lattice size reduces the power of the test, which nevertheless remains high in a 12 by 30 lattice (approximately 90\%). Concerning the robustness of the test to violations of its two basic assumptions, the method is not robust to the simulated border-effect because the type I error grows from 5 to $>15 \%$. Conversely, the dependence between $t_{1}$ cases and missing plants (2\% located at random) has no major effect (Table 4).

ESFY data set. The preliminary analysis using 2DCORR indicated that neither $t_{1}$ cases nor $t_{2}$ cases significantly differed from a random pattern, even without applying the conservative Bonferroni correction. The cumulative probability function almost perfectly matched what would be expected if diseased plants were located at random (data not shown). Consequently, in order to analyze the dependence between early and late infections, we performed Test 1. This bilateral test, with a global 5\% significance level and 1,000 simulations of $\mathrm{H}_{0}$, indicated a trend toward intra-row aggregation between $t_{1}$ cases and $t_{2}$ cases $(P=0.084$ within a distance of two trees) (Fig. 3A).

PPV data set. The preliminary analysis indicated that both $t_{1}$ cases and $t_{2}$ cases were significantly clustered (with a $P$ value below the Bonferroni threshold) for the first distance class along rows for $t_{1}$ cases, and for the first distance class along and across rows for $t_{2}$ cases. This was confirmed by a formal KolmogorovSmirnov test for $t_{2}$ cases (significant at $P<0.05$ ). Hence, to analyze the dependence between early and late infections, we used Test 3. This bilateral test with a global $5 \%$ significance level and 663 simulations of $\mathrm{H}_{0}$ indicated that $t_{1}$ cases and $t_{2}$ cases were

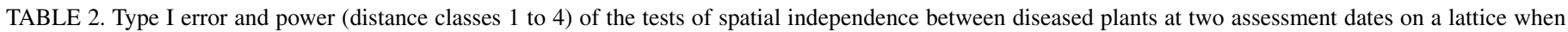
the patterns at both dates are either random or clustered, for three levels of disease incidence

\begin{tabular}{|c|c|c|c|c|c|c|c|c|}
\hline \multirow[b]{2}{*}{ Disease incidence } & \multicolumn{2}{|c|}{ Distance class 1} & \multicolumn{2}{|c|}{ Distance class 2} & \multicolumn{2}{|c|}{ Distance class 3} & \multicolumn{2}{|c|}{ Distance class 4} \\
\hline & Independent $^{\mathrm{a}}$ & Dependent $^{b}$ & Independent & Dependent & Independent & Dependent & Independent & Dependent \\
\hline \multicolumn{9}{|l|}{ Random patterns } \\
\hline $3 \%$ & 0.025 & 0.922 & 0.025 & 0.999 & 0.034 & 0.984 & 0.039 & 0.894 \\
\hline $14 \%$ & 0.037 & 0.999 & 0.052 & 1.000 & 0.038 & 0.991 & 0.049 & 0.880 \\
\hline $25 \%$ & 0.039 & 0.997 & 0.046 & 1.000 & 0.047 & 0.977 & 0.046 & 0.815 \\
\hline \multicolumn{9}{|l|}{ Clustered patterns } \\
\hline $3 \%$ & 0.022 & 0.829 & 0.032 & 0.945 & 0.036 & 0.946 & 0.037 & 0.926 \\
\hline $14 \%$ & 0.030 & 0.999 & 0.039 & 1.000 & 0.051 & 0.999 & 0.046 & 0.997 \\
\hline $25 \%$ & 0.040 & 1.000 & 0.049 & 1.000 & 0.052 & 1.000 & 0.051 & 1.000 \\
\hline
\end{tabular}

a The type I error is the probability to wrongly reject the (null) hypothesis of independence when independent patterns are simulated.

$\mathrm{b}$ The power of the test is the probability to correctly reject the (null) hypothesis of independence when dependent patterns are simulated.

TABLE 3. Type I error and power of the test of spatial independence between clusters of diseased plants at two assessment dates on a lattice, in relation to the proportion of missing plants and lattice size

\begin{tabular}{|c|c|c|c|c|c|c|c|c|}
\hline \multirow[b]{2}{*}{ Features of the data } & \multicolumn{2}{|c|}{ Distance class 1} & \multicolumn{2}{|c|}{ Distance class 2} & \multicolumn{2}{|c|}{ Distance class 3} & \multicolumn{2}{|c|}{ Distance class 4} \\
\hline & Independent ${ }^{\mathrm{a}}$ & Dependent $^{\mathrm{b}}$ & Independent & Dependent & Independent & Dependent & Independent & Dependent \\
\hline \multicolumn{9}{|c|}{ Proportion of missing plants } \\
\hline $10 \%$ & 0.034 & 0.999 & 0.037 & 1.000 & 0.046 & 1.000 & 0.040 & 0.999 \\
\hline \multicolumn{9}{|l|}{ Lattice size } \\
\hline $12 \times 30$ & 0.026 & 0.818 & 0.044 & 0.893 & 0.041 & 0.796 & 0.027 & 0.631 \\
\hline
\end{tabular}

${ }^{a}$ The type I error is the probability to wrongly reject the (null) hypothesis of independence when independent patterns are simulated.

$\mathrm{b}$ The power of the test is the probability to correctly reject the (null) hypothesis of independence when dependent patterns are simulated.

TABLE 4. Type I error and power of the test of spatial independence between clusters of diseased plants at two assessment dates on a lattice, in relation to a departure from the assumptions of stationarity and independence of missing plants

\begin{tabular}{|c|c|c|c|c|c|c|c|c|}
\hline \multirow[b]{2}{*}{ Type of departure } & \multicolumn{2}{|c|}{ Distance class 1} & \multicolumn{2}{|c|}{ Distance class 2} & \multicolumn{2}{|c|}{ Distance class 3} & \multicolumn{2}{|c|}{ Distance class 4} \\
\hline & Independent $\mathrm{t}^{\mathrm{a}}$ & Dependent ${ }^{\mathrm{b}}$ & Independent & Dependent & Independent & Dependent & Independent & Dependent \\
\hline Border effect ${ }^{c}$ & 0.135 & 1.000 & 0.146 & 1.000 & 0.161 & 1.000 & 0.151 & 1.000 \\
\hline $\begin{array}{l}\text { Aggregation with } \\
\text { missing plants }\end{array}$ & 0.035 & 0.994 & 0.052 & 0.998 & 0.048 & 1.000 & 0.044 & 0.996 \\
\hline
\end{tabular}

a The type I error is the probability to wrongly reject the (null) hypothesis of independence when independent patterns are simulated.

${ }^{b}$ The power of the test is the probability to correctly reject the (null) hypothesis of independence when dependent patterns are simulated.

${ }^{c}$ The simulated pattern is inhomogeneous, with an excessive number of diseased plants near one border of the plot.

d Missing plants are simulated at random locations, and diseased plants at time 1 are simulated around them. 
tightly aggregated, with $P$ values ranging from $P=0.012$ to 0.045 up to a distance of $10 \mathrm{~m}$ (Fig. 3B).

\section{DISCUSSION}

The analysis of epidemics simultaneously in space and time can provide crucial indications about the process of disease spread. Up to now, however, we were lacking a nonparametric method to handle disease clustering at each date and disease censoring by $t_{1}$ diseased plants and by missing plants. This has hampered the initial exploration of spatiotemporal data when plants are regularly spaced and mapped individually. In this article, we have presented a framework to test the hypothesis that the location of newly diseased plants is independent of the location of previously diseased plants. In this permutation method dedicated to the exploration of spatiotemporal data, the significant spatial structures at each date and the censoring are taken into account. This method can provide indications on the role of short-distance plant-to-plant transmission, which is fundamental for both epidemiological studies and disease management.

The validation of our method on numerous simulated bivariate point patterns shows that it is powerful in the detection of dependent patterns (even for a relatively small lattice size such as 12 by 30 ). It also shows that, when independent patterns are simulated, the rate of rejection of the hypothesis of independence is around the predefined 5\% significance level, or slightly below for small lattices. Of course, the accuracy and power of the method could be assessed for other combinations of the parameters that define the processes; the properties of the test in any specific situation can be studied numerically as exemplified in this article, by simulating the appropriate patterns.

The analysis of data sets from ESFY and PPV epidemics demonstrates the practical application of this method that allowed testing general spatiotemporal hypotheses related to the processes of disease spread for both diseases. For example, as the epidemiology of ESFY remains poorly characterized, secondary transmission at the orchard scale is still an open question. The test of independence indicated a trend toward within-row aggregation between the trees showing symptoms early in the epidemic and those developing disease later; this trend is consistent with short-range intra-orchard transmission. However, the same analyses should be performed on several representative orchards to confirm that this trend is, in fact, a general feature of the spread of ESFY.

The second example addressed the spatiotemporal spread of PPV-M in a peach orchard. Natural epidemics caused by the PPV$M$ strain frequently result in highly clustered patterns of disease (9). However, when disease management is strict within the orchard (systematic visual identification and roguing of symptomatic trees) and immediately effective, there should be no spatial dependence between new (exogenous) infections and previously diseased trees. Thus, analyzing the distances between diseased trees that have been rogued and trees that showed symptoms the year following the removal can provide clues about the effectiveness of this control method. As the aggregation between newly and previously symptomatic plants was very strong, it appears that the continuing progress of the disease within this orchard was principally driven by internal processes, despite the implemented control method.

The analysis of epidemiological data goes from an acute observation of disease patterns to a comprehensive understanding of epidemiological processes. Among the corresponding approaches that are currently used to study spatiotemporal data, our tests occupy an intermediate position between methods that provide a detailed summary of the spatiotemporal patterns (28) and those for estimating parameters of disease spread (15). Testing independence of diseased plants between two dates is attractive because this hypothesis is linked to the process of disease spread, and it naturally associates spatial and temporal patterns. Thus, such tests complement the approaches that provide precise descriptions of the patterns through the empirical comparison of spatial analyses at successive dates (17) or through the separate analysis of the temporal and spatial features of a disease $(2,37,38)$. When the plants are mapped individually as healthy or diseased, analyses based on point patterns use more information than quadrat-based techniques and usually provide a more detailed insight into the data. Conversely, if disease notation is quantitative or if the health
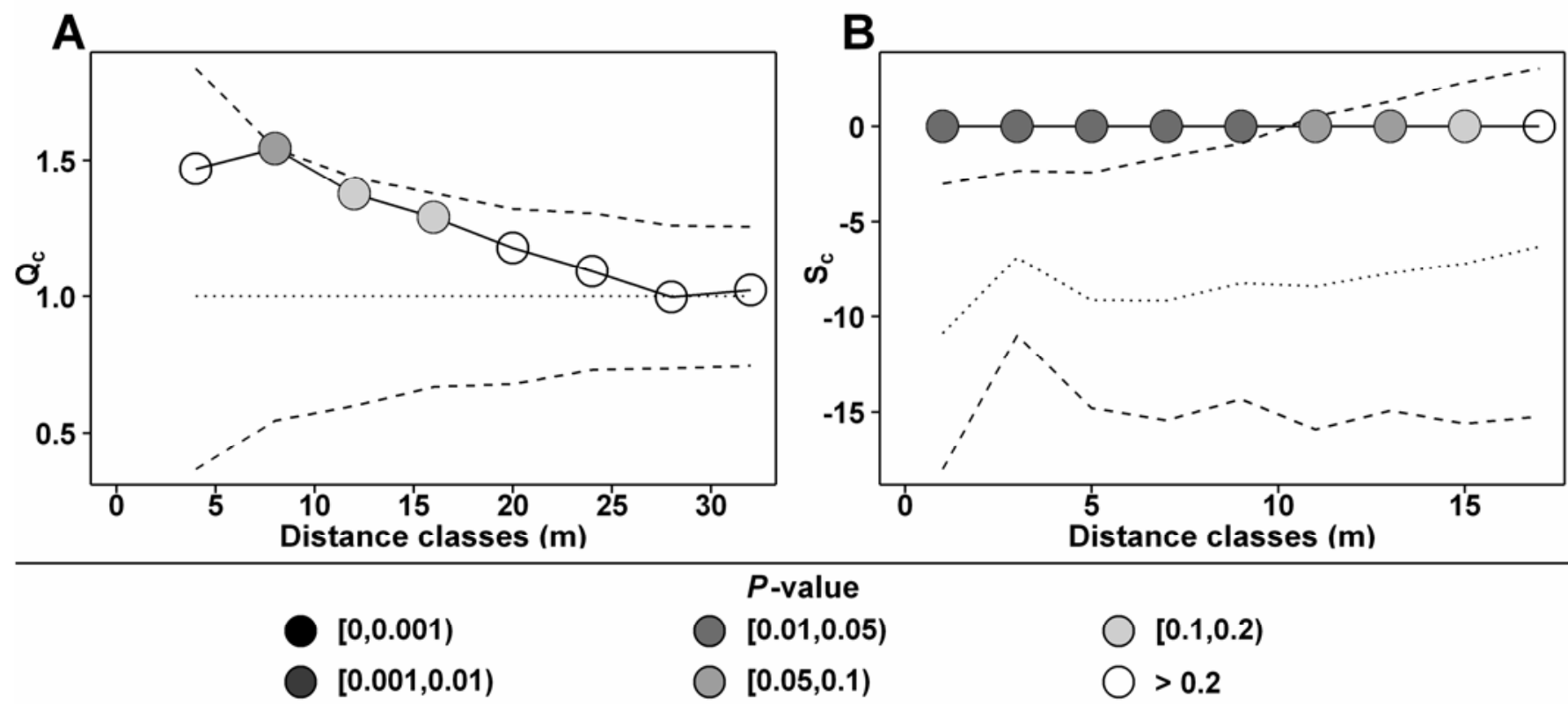

$[0.1,0.2)$

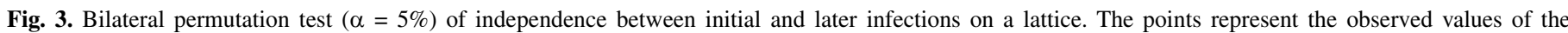

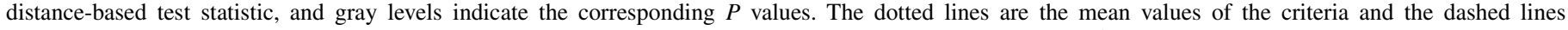

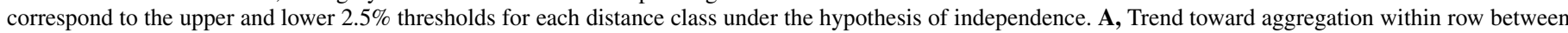

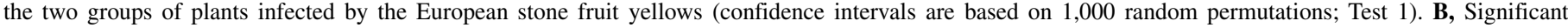

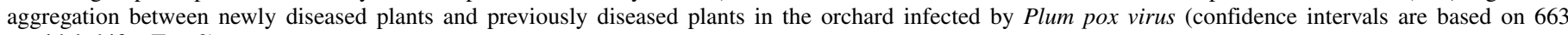
toroidal shifts; Test 3). 
status is assessed for a group of plants, an analysis with other methods like spatiotemporal autocorrelation analysis $(8,35)$ or other quadrat-based methods (5) could be more appropriate. Our method is suitable to analyze poorly known diseases, because it makes no assumption about unknown proximity patterns or distribution functions. Hence, distribution-free hypothesis tests can play a role in the construction of mechanistic models, as they can be used in the initial steps of modeling, when one has to decide which basic mechanisms and assumptions have to be included. The test statistic $Q_{c}(d)$ can also be used to assess the fit of a stochastic model to a data set, through a Monte Carlo test where the confidence envelopes are defined by repeated realizations of the model and computation of the corresponding values of $Q_{c}(d)$. When sufficient information is available on the basic processes of spread and on the distribution of the associated parameters, parametric analysis and more specifically mechanistic modeling is probably the most powerful approach to gain insights into disease spread and to estimate epidemiological parameters.

In addition to its contribution to plant disease epidemiology, our method can also be adapted to analyze spatiotemporal data or censored spatial data in ecology. A test using random shifts to investigate the spatial dependence between two point patterns given the spatial structure within each group of points has already been described in a continuous space $(10,22)$ and used in spatial ecology $(1,11,14,16,31)$, mainly to study the interactions between two populations (competition or facilitation). In continuous space, random shifts of points do not generate censoring, because the probability of a point being shifted to the exact position of another point is null. In contrast, when plants are grown in a regular lattice, simulated points can censor actual points so these events had to be taken into account. Despite this fundamental difference, our tests might be applied to cope with censored data in the analysis of ecological data in which no plant can grow in a portion of the region under study (e.g., rocky or flooded zones) while the remainder of the area is homogenous. In combination with the approach developed by Pélissier and Goreaud (30) to determine the limits of such inhospitable areas, the method could provide a way to test the interactions between two populations. Moreover, Test 1 and Test 2 can be applied directly to irregular plantings, so they can be used to analyze disease spread between two dates in natural landscapes and in rows of heterogeneous density. Of course, the method can also be adapted if one is interested in the proximity pattern between events that do not cause censoring, i.e., in which the two events can be observed simultaneously (e.g., two diseases causing discernable symptoms on the same plant, or two insect species). Besides these modifications that could broaden the application of this method, we identified some limitations that can be the starting point for future work, which could lead to more sophisticated analyses of spatiotemporal data.

In common with many methods of spatial analysis, the tests described in this paper require a few assumptions; it is necessary to specify them and to point out their implications for the interpretation of the results. First, we assume that missing plants are independent of disease, so the demonstration of their spatial dependence modifies the interpretation. For example, if missing plants are associated with $t_{1}$ cases, depending on the objectives it might be relevant to consider them as early diseased plants and to incorporate them into $t_{1}$ cases. However, at least when few missing plants are located at random, the dependence between $t_{1}$ cases and missing plants has no major impact on the test (Table 4).

The second assumption is that the process is stationary (i.e., when shifted, its statistical properties are invariant). When the preliminary analysis shows a significant border-effect in disease incidence, our tests would often detect a nonrandom association between the two dates (Table 4). The observed border-effect is an obvious cause to this association, which can hide other potential sources of dependence that are more interesting in understanding disease spread. Thus, for nonstationary processes, our test should not be used as is: the null hypothesis should include any existing border-effect, and more specific tests will be required. Similarly, any underlying structure in the field that is related to the disease (e.g., heterogeneous soil, sowing date, cultivar, etc.) violates the assumption of stationarity. In these situations, random permutations and shifts should be performed within each stationary subset; afterwards, the distances can be combined in a global test.

Toroidal distances in Test 3 artificially group together distant points located near the two vertical or horizontal edges of the plot. Thus, Test 3 is more suitable when the size of the lattice widens, because the weight of these edge-effects in the analysis decreases. It is noteworthy that Test 3 computes all distances on a torus, with the result that observed and simulated patterns are consistent: on a torus, the distribution of distances within a shifted group of points does not differ from the distances within the same group before shift. Hence, when we simulate independent patterns on a small lattice $(12 \times 30)$, the type I error is still around $5 \%$ (Table 3 ). The way the censoring is handled in Test 2 and Test 3 induces a drawback: the exclusion of part of the data slightly reduces the power of these tests. A solution to both issues (the use of toroidal shifts and the handling of censored data) is to identify the point process that generates the observed pattern of $t_{2}$ cases and to simulate the null hypothesis according to this point process (16). However, identifying a point process from a unique censored realization is a difficult task because different processes can produce the same pattern.

Further development could improve some aspects of the tests. For example, currently, we only seek directional aggregation (or repulsion) along rows or across rows. Yet a major benefit of twodimensional distance class analysis comes from its ability to detect a directional spread of diseases potentially driven by wind or by plant contacts $(12,13,19)$. So, additional work could intend to allow the detection of wind-driven spread or to incorporate the tests in a two-dimensional framework. It is possible to generate a map-like output because noncumulative versions of $Q_{c}(d)$ and $S_{c}(d)$ can be computed for each distance-orientation class. However, when the phenomenon under study is isotropic, splitting distance classes into distance-orientation classes lowers the statistical power because of the decrease in the number of pairs of plants in each class. The resulting lack of statistical power, in combination with the problem of multiple testing (12) restricts the use of such a two-dimensional analysis to diagnostic purposes, in the exploratory phase of studies. As shown by Ferrandino (13), the two approaches could be combined for providing both a twodimensional insight into the phenomenon (with limited statistical power) and a nondirectional test based on plants less than a distance apart from each plant (with better statistical properties), like $Q_{c}(d)$ and $S_{c}(d)$.

Another feature of our method is that in contrast to other spatiotemporal methods $(15,35)$, generalizing the method to more than two dates implies a clear reassessment of the hypothesis that one wants to test. In its present form, even when three or more dates are available, the test of independence only uses two dates or two groups of dates. However, several assessment dates can be combined, for example on the basis of the incubation period if it is longer than the time interval between two successive visual assessments. Another possibility is that several paired dates or groups of dates can be analyzed in an exploratory fashion. However, such a choice reduces the statistical value of the tests if they are not corrected to take account of multiple testing (12), because it increases the risk to detect a spurious significant dependence. Thus, in order to limit the number of tests, the hypothesis should be clearly stated (e.g., the pattern at each date is independent of the pattern at the date before) and groups should be decided $a$ priori. However, to detect a general trend in a spatiotemporal data set, it should be possible to build a global test that synthesizes the distances computed on several independent pairs of dates. 
In this article, we have developed a versatile framework for testing hypotheses on the independence of the positions of diseased plants at two dates, even when there is censoring and spatial dependence within each group of plants. The method can be used as described, or adapted to test the spatial dependence between two groups of points in other systems. By testing hypotheses of independence, our aim is to make inferences about the biological processes of disease spread, in the line of the position promoted by Hughes and Madden (20): "Ultimately it will be desirable to forge links between statistical descriptions of spatial patterns of plant disease and observations and theories about the dispersal of plant pathogens and disease vectors." However, such links are indirect as we do not test the biological mechanism itself but its consequence, we obtain clues rather than a definitive demonstration of the biological processes (10). Thus, the demonstration of dependence between two dates should not be interpreted in terms of direct transmission without considering the competing explanations (e.g., in relation to the latent period, the infectious period, and the susceptibility of the plants). Therefore, in order to unveil major features of spatiotemporal data sets and to select the most likely explanatory processes, we suggest to use the method described here as one element in a broader exploratory approach rather than as a unique ready-made tool.

Successive hypothesis tests can be used because their flexibility allows progressive refinements of the initial hypothesis. Thus, testing independence between two disease assessment dates becomes one step in a more general question-oriented strategy. It is also possible to combine the results of several complementary methods dedicated to the analysis of disease incidence data in time or space $(18,37)$. These multiple analyses provide a nuanced perception of disease progress: they allow testing of hypothetical processes and they are a basis to develop new hypotheses. Furthermore, the best way to get a comprehensive view of an epidemic is to use an integrated approach including spatiotemporal data analyses alongside experimental studies.

\section{APPENDIX}

Test 2. This section corresponds to the theory for the permutation test when the diseased plants at date 2 show a spatial structure whereas the diseased plants at date 1 are not structured. $Z_{1}$ and $Z_{2}$ denote the corresponding underlying point processes, which are partly censored by an independent censoring point pattern $X$ corresponding to missing plants. In this situation, the positions of observed $t_{1}$ cases $\left(Z_{1} \cap \bar{X}\right)$ are independent and identically distributed (i.e., at random). The tested hypothesis $\left(\mathrm{H}_{0}\right)$ is as follows: "the two point processes $Z_{1}$ and $Z_{2}$ are independent."

$Y_{1}=Z_{1} \cap \bar{X}$ and $Y_{2}=Z_{2} \cap \bar{X}$ are the subsets of diseased plants that are not censored by $X$ at dates 1 and 2, respectively. Since $Z_{1}$ and $Z_{2}$ are independent (under $\mathrm{H}_{0}$ ) and are independent of $X, Z_{1} \mid X$ is independent of $Z_{2} \mid X$. This implies that $Y_{1} \mid X$ is independent of $Y_{2} \mid X$.

Let $Y_{1}^{\phi}$ be the result of a permutation $\phi$ of $Y_{1}$ (on $\bar{X}$ ). If $L$ denotes the probability law, $L\left(Y_{1} \mid X\right)=L\left(Y_{1}^{\phi} \mid X\right)$. This fact, together with the independence between $Y_{1} \mid X$ and $Y_{2} \mid X$, leads to $L\left(Y_{1}, Y_{1}^{\phi}, Y_{2} \mid X\right)=L\left(Y_{1}^{\phi}, Y_{1}, Y_{2} \mid X\right)$. Then, for any transformation $f$, $L\left[f\left(Y_{1}, Y_{1}^{\phi}, Y_{2}\right) \mid X\right]=L\left[f\left(Y_{1}^{\phi}, Y_{1}, Y_{2}\right) \mid X\right]$. The case

$$
f(u, v, w)=(w \cap \bar{u} \cap \bar{v}, u)
$$

yields:

$$
L\left(Y_{2} \cap \overline{Y_{1}} \cap \overline{Y_{1}^{\phi}}, Y_{1} \mid X\right)=L\left(Y_{2} \cap \overline{Y_{1}} \cap \overline{Y_{1}^{\phi}}, Y_{1}^{\phi} \mid X\right) .
$$

With the simplified notations used in the text, this can be written $L\left(R_{2}, Y_{1}\right)=L\left(R_{2}, Y_{1}^{\phi}\right)$ : the distribution of the distances between observed $t_{1}$ cases and observed $t_{2}$ cases that are not overlaid by a $t_{1}$ case after permutation is the same as the distribution of distances between $t_{1}$ cases after permutation and observed $t_{2}$ cases that are not overlaid by a $t_{1}$ case after permutation.
Test 3. The demonstration corresponding to Test 3 (to be applied when there is a significant spatial structure at both dates) is more straightforward. We assume that $Z_{1}$ and $Z_{2}$ are independent of the external censoring $X$ (missing plants), that the point processes are stationary and invariant by toroidal shifts. The tested hypothesis $\left(\mathrm{H}_{0}\right)$ is as follows: "the two point processes $Z_{1}$ and $Z_{2}$ are independent." Under these assumptions and for a given toroidal shift $\phi, Z_{2}$ is independent of $Z_{1}, X, Z_{1} \cup Z_{1}^{\phi}$, and $X \cup X^{\phi}$. Thus, their joint probability law is invariant for any independent transformation of $Z_{2}$.

Applying $\phi$ to $Z_{2}$ leads to:

$$
L\left(Z_{2}, Z_{1}, X, Z_{1} \cup Z_{1}^{\phi}, X \cup X^{\phi}\right)=L\left(Z_{2}^{\phi}, Z_{1}, X, Z_{1} \cup Z_{1}^{\phi}, X \cup X^{\phi}\right) .
$$

Hence, for any transformation $f$ :

$$
L\left[f\left(Z_{2}, Z_{1}, X, Z_{1} \cup Z_{1}^{\phi}, X \cup X^{\phi}\right)\right]=L\left[f\left(Z_{2}^{\phi}, Z_{1}, X, Z_{1} \cup Z_{1}^{\phi}, X \cup X^{\phi}\right)\right] .
$$

The case $f(u, v, w, x, y)=(v \cap \bar{w}, u \cap \bar{x} \cap \bar{y})$ leads to:

$L\left[Z_{1} \cap \bar{X}, Z_{2} \cap\left(\overline{Z_{1} \cup Z_{1}{ }^{\phi}}\right) \cap\left(\overline{X \cup X^{\phi}}\right)\right]=L\left[Z_{1} \cap \bar{X}, Z_{2}^{\phi} \cap\left(\overline{Z_{1} \cup Z_{1}{ }^{\phi}}\right) \cap\left(\overline{X \cup X^{\phi}}\right)\right]$.

With the simplified notations used in the text, this can be written $L\left(Y_{1}, R_{2}^{\prime}\right)=L\left(Y_{1}, R_{2}^{\prime}{ }^{\phi}\right)$ : the distribution of the distances between observed $t_{1}$ cases and observed $t_{2}$ cases that are not overlaid by a shifted $t_{1}$ case or a shifted missing plant is the same as the distribution of distances between observed $t_{1}$ cases and shifted $t_{2}$ cases that are not overlaid by a $t_{1}$ case or a missing plant.

\section{ACKNOWLEDGMENTS}

We thank E. Klein for fruitful discussions during the preparation of the manuscript, C. Bock for correcting a previous version of the text, and C. Castelain and the Fédération Régionale de Défense contre les Organismes Nuisibles (FREDON) of Languedoc-Roussillon for providing the two disease maps used in this paper.

\section{LITERATURE CITED}

1. Barot, S., Gignoux, J., and Menaut, J. C. 1999. Demography of a savanna palm tree: Predictions from comprehensive spatial pattern analyses. Ecology 80:1987-2005.

2. Bassanezi, R. B., Bergamin, A., Amorim, L., Gimenes-Fernandes, N., Gottwald, T. R., and Bové, J.-M. 2003. Spatial and temporal analyses of citrus sudden death as a tool to generate hypotheses concerning its etiology. Phytopathology 93:502-512.

3. Besag, J. E. 1977. Discussion on Dr. Ripley's paper. J. R. Stat. Soc. B 39:193-195.

4. Campbell, C. L. 1986. Interpretation and uses of disease progress curves for root diseases. Pages 38-54 in: Plant Disease Epidemiology, vol. 1: Population Dynamics and Management. K. J. Leonard and W. E. Fry, eds. Macmillan, New York.

5. Campbell, C. L., and Madden, L. V. 1990. Introduction to Plant Disease Epidemiology. Wiley, New York.

6. Chadœuf, J., Brix, A., Pierret, A., and Allard, D. 2000. Testing local dependence of spatial structures on images. J. Microsc. 200:32-41.

7. Chadœuf, J., Capowiez, Y., Kretzschmar, A., and Dessart, H. 1997. Testing interaction between a random area process and another spatial process: The analysis of spatial patterns of soil sections. Acta Stereol. 16:251-258.

8. Cliff, A. D., and Ord, J. K. 1981. Spatial Processes: Models and Applications. Pion Ltd., London.

9. Dallot, S., Gottwald, T., Labonne, G., and Quiot, J.-B. 2003. Spatial pattern analysis of sharka disease (Plum pox virus strain $\mathrm{M}$ ) in peach orchards of southern France. Phytopathology 93:1543-1552.

10. Diggle, P. J. 1983. Statistical Analysis of Spatial Point Patterns. Academic Press, London.

11. Duncan, R. P. 1991. Competition and the coexistence of species in a mixed podocarp stand. J. Ecol. 79:1073-1084.

12. Ferrandino, F. J. 1996. Two-dimensional distance class analysis of disease-incidence data: Problems and possible solutions. Phytopathology 86:685-691.

13. Ferrandino, F. J. 1998. Past nonrandomness and aggregation to spatial correlation: 2DCORR, a new approach for discrete data. Phytopathology 88:84-91.

14. Forget, P. M., Mercier, F., and Collinet, F. 1999. Spatial patterns of two rodent-dispersed rain forest trees Carapa procera (Meliaceae) and Vouacapoua americana (Caesalpiniaceae) at Paracou, French Guiana. J. Trop. Ecol. 15:301-313. 
15. Gibson, G. J., Kleczkowski, A., and Gilligan, C. A. 2004. Bayesian analysis of botanical epidemics using stochastic compartmental models. Proc. Natl. Acad. Sci. USA 101:12120-12124.

16. Goreaud, F., and Pélissier, R. 2003. Avoiding misinterpretation of biotic interactions with the intertype $\mathrm{K}_{12}$-function: Population independence vs. random labelling hypotheses. J. Veg. Sci. 14:681-692.

17. Gottwald, T. R., Avinent, L., Llacer, G., Hermoso de Mendoza, A., and Cambra, M. 1995. Analysis of the spatial spread of sharka (plum pox virus) in apricot and peach orchards in eastern Spain. Plant Dis. 79:266278.

18. Gottwald, T. R., Cambra, M., Moreno, P., Camarasa, E., and Piquer, J. 1996. Spatial and temporal analyses of Citrus tristeza virus in eastern Spain. Phytopathology 86:45-55.

19. Gray, S. M., Moyer, J. W., and Bloomfield, P. 1986. Two-dimensional distance class model for quantitative description of virus-infected plant distribution lattices. Phytopathology 76:243-248.

20. Hughes, G., and Madden, L. V. 1992. Aggregation and incidence of disease. Plant Pathol. 41:657-660.

21. Hughes, G., McRoberts, N., Madden, L. V., and Gottwald, T. R. 1997. Relationships between disease incidence at two levels in a spatial hierarchy. Phytopathology 87:542-550.

22. Lotwick, H. W., and Silverman, B. W. 1982. Methods for analyzing spatial processes of several types of points. J. R. Stat. Soc. B 44:406-413.

23. Madden, L. V., and Hughes, G. 1999. Sampling for plant disease incidence. Phytopathology 89:1088-1103.

24. Manly, B. F. J. 1991. Randomization and Monte Carlo Methods in Biology. Chapman \& Hall, London.

25. McRoberts, N., Hughes, G., and Madden, L. V. 1996. Incorporating spatial variability into simple disease progress models for crop pathogens. Aspects Appl. Biol. 46:75-82.

26. Mugglestone, M. A., Lee, B. Y. Y., Roff, M. N. M., Jones, P., Plumb, R. T., and Deadman, M. L. 1996. Point process modelling of BYMV incidence in lupins. Aspects Appl. Biol. 46:87-94.

27. Nelson, S. C. 1995. STCLASS-Spatiotemporal distance class analysis software for the personal computer. Plant Dis. 79:643-648.

28. Nelson, S. C. 1995. Spatiotemporal distance class analysis of plant disease epidemics. Phytopathology 85:37-43.
29. Nelson, S. C., Marsh, P. L., and Campbell, C. L. 1992. 2DCLASS, a twodimensional distance class analysis software for the personal computer. Plant Dis. 76:427-432.

30. Pélissier, R., and Goreaud, F. 2001. A practical approach to the study of spatial structure in simple cases of heterogeneous vegetation. J. Veg. Sci. 12:99-108.

31. Penttinen, A., Stoyan, D., and Henttonen, H. M. 1992. Marked pointprocesses in forest statistics. Forest Sci. 38:806-824.

32. Pethybridge, S. J., Wilson, C. R., Ferrandino, F. J., and Leggett, G. W. 2000. Spatial analyses of viral epidemics in Australian hop gardens: Implications for mechanisms of spread. Plant Dis. 84:513-515.

33. Peyrard, N., Calonnec, A., Bonnot, F., and Chadœuf, J. 2005. Explorer un jeu de données sur grille par tests de permutation. Rev. Stat. Appl. LIII:59-78.

34. R Development Core Team. 2004. R: A Language and Environment for Statistical Computing. R Foundation for Statistical Computing, Vienna, Austria.

35. Reynolds, K. M., and Madden, L. V. 1988. Analysis of epidemics using spatio-temporal autocorrelation. Phytopathology 78:240-246.

36. Ripley, B. D. 1977. Modelling spatial patterns. J. R. Stat. Soc. B 39:172 212.

37. Roumagnac, P., Pruvost, O., Chiroleu, F., and Hughes, G. 2004. Spatial and temporal analyses of bacterial blight of onion caused by Xanthomonas axonopodis pv. allii. Phytopathology 94:138-146.

38. Smith, M. C., Page, W. W., Holt, J., and Kyetere, D. 2000. Spatial dynamics of maize streak virus disease epidemic development in maize fields. Int. J. Pest Manage. 46:55-66.

39. Thébaud, G., Labonne, G., Castelain, C., and Chadœuf, J. 2004. Spatiotemporal analysis of disease spread provides insights into the epidemiology of European stone fruit yellows. Acta Hortic. 657:471-476.

40. Upton, G., and Fingleton, B. 1985. Spatial Data Analysis by Example. vol. 1: Point Pattern and Quantitative Data. Wiley, Chichester.

41. Vanderplank, J. E. 1963. Plant Diseases: Epidemics and Control. Academic Press, New York.

42. Waggoner, P. E., and Rich, S. 1981. Lesion distribution, multiple infection, and the logistic increase of plant disease. Proc. Natl. Acad. Sci. USA 78:3292-3295. 